\title{
The COVID-19 Era: Myths and Realities in the Ethiopian Context
}

\section{Shimels T*}

Saint Paul's Hospital Millennium Medical College, Ethiopia

*Corresponding author: Tariku Shimels, Lecturer (Research coordinator), Saint Paul's Hospital Millennium Medical College, Addis Ababa, Ethiopia, Email: tarphar2008@gmail.com

\section{Mini Review \\ Volume 5 Issue 1}

Received Date: December 31, 2020

Published Date: January 12, 2021

DOI: $10.23880 /$ phoa- 16000171

\section{Abstract}

Coronavirus disease-2019 (COVID-19) is a viral infection caused by the new strain of Coronavirus called severe acute respiratory syndrome coronavirus 2 (SARS-COV2) originated in December 2019 at the Chinese city of Wuhan. Since then, it has spread globally at an unprecedented scale and infected about 77,530,799 people, and killed 1,724,904 at its one year course. In Ethiopia, there have been 120,638 confirmed cases of COVID-19 with 1,864 deaths on the same date. Trend on number of cases and deaths showed a remarkable seasonal rise blown since the start of summer with varying, but high number of attacks until to date. Following the WHO's marking of COVID-19 as global public health threat, countries had placed diverse strategies to prevent manage the outbreak most of which are still on implementation. Following identification of the first confirmed case in March, the Ethiopian government placed multiple measures through a state of emergency to prevent the transmission of the virus. These included; lockdown, social distancing, hand washing practices, use of a sanitizer, wearing of face masks, limited number of people on public transportation loads, prohibiting public (mass) assemblies, and stay at home campaigns among others. Despite all these efforts, there was a considerable gap to retain an optimum practice of prevention methods attributed to a range of reasons. This, in turn, is leading to an increasing number of cases and mortalities over time. In this commentary, I forward my own reflection and observation in relation to common myths held among some opinion leaders and a significant segment of the society, which I believe, has impacted the way COVID-19 is evolving now. The myths will also be discussed in light with available facts documented in the literature.

Keywords: Myths and Realities; COVID-19; Public Health; Sanitizer

\section{Myths and Realities about the Pandemic and its Treatment Modalities}

\section{Belief that COVID-19 Worsens with Racial Vulnerability}

Despite acceptance of COVID-19's viral causality among this group, there is a widespread opinion that the disease would not be as virulent among blacks as it would be to the Whites. Some try to justify this belief by comparing observed figures in developed and developing nations, especially, in the sub-Saharan. Some argue that immunity level may vary by race dramatically which might put blacks at a longer experience to live with, fight against, and survive from infectious agents [1-3]. They say because viral infections and other respiratory tract infections are prevalent in the
sub-Saharan, people of this region can easily cope up with virus. This opinion, however, can be challenged or supported in different arguments. While spread of the virus is steadily growing over time, evidences also show that blacks had greater risk of acquiring the infection or being admitted or died $[4,5]$. The risk is estimated to be higher among those with Asian origin [4]. Yet, most of the studies reporting such figures were from either the United States (US) or the United Kingdom (UK) which may not present the actual scenario in Africa or other part of the developing world. The seemingly paradoxical low mortality rate in Africa is reported to be low life expectancy, lower population mean age, lower preCOVID-19 era '+65years death rate, and small number of people living with and surviving from cardiovascular disease [6]. On the reverse, some hypotheses also hold that the low morbidity and mortality in Africa could be due to low seeding 
rate, effective mitigation measures, and population that is more youthful, favorable weather, and possible preexisting immunity due to prior exposure to other coronaviruses [7]. Deduced from the assumptions in noted in the sub-Sahara region, the situation in Ethiopia follows almost a similar fashion. The curve remains to reveal an upward trend with noticeable peak during humid seasons.

\section{Belief that SARS-COV2 is Released from Lab}

Even at today, there is a strong share of thinking, by many people in Ethiopia, that the SARS-COV2 virus is produced in the laboratory. Despite unavailability of concrete evidence, some elites argue that COVID-19 is a disease due to a variant of already known clusters coronavirus family. They claim it is manipulated through a high tech genetic engineering. They also ascribe their justification by relating the sign and symptoms as well as prognosis of the disease with earlier documentaries, such as 'The Contagion (2011)'. Studies have tried to put a scientific explanation on the improbability that SARS-CoV-2 emerged through laboratory manipulation of a related SARS-CoV-like coronavirus. Rather, a study proposed two scenarios as (a); natural selection in an animal host before zoonotic transfer; and (b) natural selection in humans following zoonotic transfer [8]. Another study by Bolsen and his colleagues [9] on a US population tested four hypotheses namely; natural origins of the virus, one manipulated in the lab, impact of conspiracy belief penalize China or support increased funding for biomedical research. The study showed that framing of origin information either to the natural or China conspiracy plays a vital role where both theories showed statistically significant account in the respective group. Likely, the impact of 'origin belief ' evaluation in the same study showed that people with a belief that the virus was engineered and leaked from lab were more willing to penalize China versus those who believe the virus was of natural origin opted to support for further biomedical researches.

\section{Facts to Note}

Poor practice of preventive measures and large scale of public ignorance might be a risk that leads to extended virus transmission in most settings. Even law enforcement bodies do not take stringent actions in some pubic recreational domains, such as groceries, bars, hotels and cafeteria, transportation places and privately owned higher education institutions. It is evident that number of cases in the country surge steadily overtime despite a seemingly lower rate compared to developed nations. Comparisons and small numbers should not be a relief against plausible high morbidity and mortality outcomes. Increasing trend of numbers should be seen in light of loose preventive strategies at all levels. The pandemic has posed a new paradigm in people's life and public health. Most schools and organizations have shifted previous practices to accord with the COVID-19 era. Multiple businesses have been created, especially, to the lower economy group of towns and cities where street business showed to avail personal protective equipment (PPE) to the lower majority. Health authorities have diverted their resources significantly to trainings, preventive actions, and treatment centers of COVID-19. New projects are arising while pre-COVID-19 ones have been closed. Yet, questions of health service quality and coverage on other communicable and non-communicable diseases have not gained emphasis as it should be. Further attention is required by all concerned stakeholders.

\section{References}

1. Zhu H, Wei L, Niu P (2020) The novel coronavirus outbreak in Wuhan, China. Glob Health Res Policy 5: 6.

2. WHO (2020) WHO Coronavirus Disease (COVID-19) Dashboard. World Health Organization.

3. WHO (2020) WHO (COVID-19) Homepage; Ethiopia Situation. World Health Organization.

4. Sze S, Pan D, Nevill CR, Gray LJ, Martin CA, et al. (2020) Ethnicity and clinical outcomes in COVID-19: A systematic review and meta-analysis. E Clinical Medicine 29: 100630 .

5. Doumas M, Patoulias D, Katsimardou A, Konstantinos S, Konstantinos I, et al. (2020) COVID19 and increased mortality in African Americans: socioeconomic differences or does the renin angiotensin system also contribute? J Hum Hypertens 34(11): 764-767.

6. Lawal Y (2021) Africa's low COVID-19 mortality rate: A paradox? Int J Infect Dis 102: 118-122.

7. Njenga MK, Dawa J, Nanyingi M, Gachohi J, Ngere I, et al. (2020) Why is There Low Morbidity and Mortality of COVID-19 in Africa? Am J Trop Med Hyg 103(2): 564569.

8. Andersen KG, Rambaut A, Lipkin WI, Edward CH, Robert FG (2020) The proximal origin of SARS-CoV-2. Nat Med 26(4): 450-452.

9. Bolsen T, Palm R, Kingsland JT (2020) Framing the Origins of COVID-19. Sci Commun 42(5): 562-585.

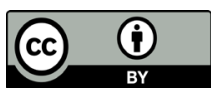

